\title{
Relating diameter and mean curvature for submanifolds of Euclidean space
}

\author{
Peter Topping
}

Abstract. Given a closed $m$-dimensional manifold $\mathcal{M}$ immersed in $\mathbb{R}^{n}$, we estimate its diameter $d$ in terms of its mean curvature $\boldsymbol{H}$ by

$$
d \leq C(m) \int_{\mathcal{M}}|\boldsymbol{H}|^{m-1} d \mu .
$$

Mathematics Subject Classification (2000). 49Q10, 53A05, 53A07, 53C20, 53C40, 53C42.

Keywords. Geometric inequalities, diameter, mean curvature, volume, Michael-Simon Sobolev inequalities, geometric maximal function.

\section{Introduction}

Given a closed connected surface $\mathcal{M}^{2}$ immersed in $\mathbb{R}^{3}$, there are a number of geometric inequalities relating fundamental quantities such as diameter, area, curvature and (when defined) enclosed volume. One particularly useful example is an inequality of Leon Simon which relates the extrinsic diameter ${ }^{1} d_{\text {ext }}:=\max _{x, y \in \mathcal{M} \hookrightarrow \mathbb{R}^{3}}|\boldsymbol{x}-\boldsymbol{y}|_{\mathbb{R}^{3}}$ to the area $A$ and mean curvature $H$ (here the average rather than the sum of the principle curvatures) by

$$
d_{\mathrm{ext}}<\frac{2}{\pi} A^{\frac{1}{2}}\left(\int_{\mathcal{M}} H^{2} d \mu\right)^{\frac{1}{2}}
$$

where $\mu$ is the measure on $\mathcal{M}$ induced by the ambient space. See [4] for the original proof and applications, [6] for the perturbed proof with the constant $\frac{2}{\pi}$ (presumably optimal up to a factor of two, by consideration of a long cylinder with capped ends) and [1] for another paper which uses the result.

\footnotetext{
${ }^{1}$ we will adopt the convention that a point $x \in \mathcal{M}$ is sent to a point $x \in \mathbb{R}^{n}$ by the immersion.
} 
It is hard to look at this estimate for long without speculating that it is, in fact, a special case of a stronger simpler estimate, and in [6] we conjectured that

$$
d_{\mathrm{ext}}<\frac{1}{\pi} \int_{\mathcal{M}}|H| d \mu
$$

to which one could apply the Cauchy-Schwarz inequality to deduce (1.1) with the (conjectured) optimal constant.

In this paper, we prove (1.2) with the constant $\frac{1}{\pi}$ weakened to $\frac{32}{\pi}$, but with the estimate strengthened by replacing the extrinsic diameter $d_{\mathrm{ext}}$ with the intrinsic diameter $d_{\text {int }}:=\max _{x, y \in \mathcal{M}} \operatorname{dist}_{\mathcal{M}}(x, y)$. We also generalise it to any dimension, and codimension.

We remark that in the case that $\mathcal{M}$ is a surface of constant mean curvature $H$ immersed in $\mathbb{R}^{3}$, we established in [5] that

$$
d_{\mathrm{ext}} \leq \frac{A|H|}{2 \pi},
$$

which is twice as strong as (1.2). Equality is then achieved when $\mathcal{M}$ is a sphere. The methods required for that are entirely different, relying on special properties of Jacobian determinants. We also point out that if one is willing to weaken the inequality (1.2) by replacing $|H|$ by the norm of the second fundamental form (and adjusting the constant $\frac{1}{\pi}$ ) then one can proceed directly by a slicing argument ([4]).

In order to work in any codimension in the following theorem, we will work now with the mean curvature vector $\boldsymbol{H}$. Our normalisation is that for the unit sphere in $\mathbb{R}^{n}$, the vector $\boldsymbol{H}$ should be the inward unit vector.

Theorem 1.1. For $n>m \geq 1$, suppose that $\mathcal{M}$ is an m-dimensional closed (compact, no boundary) connected manifold smoothly immersed in $\mathbb{R}^{n}$. Then its intrinsic diameter $d_{\text {int }}$ and its mean curvature $\boldsymbol{H}$ are related by

$$
d_{\text {int }} \leq C(m) \int_{\mathcal{M}}|\boldsymbol{H}|^{m-1} d \mu .
$$

We can take $C(2)=\frac{32}{\pi}$.

Aside from the works already cited, the closest precedent for this theorem is our work on diameter estimates for intrinsic manifolds evolving under Ricci flow [7]. In the present paper a core tool will be the Michael-Simon Sobolev inequality Lemma 2.1, originally from [2]; in the Ricci flow setting we used the direct analogue of Michael and Simon's result, which is the Ricci flow log-Sobolev inequality implied by the monotonicity of Perelman's W-entropy (see [3], [8]).

At the core of the proof of (1.1) was the following assertion that one cannot simultaneously have small area and small curvature in a ball within the surface. 
Given $x \in \mathcal{M}^{2}$, we denote the subset of $\mathcal{M}$ immersed inside the open extrinsic ball in $\mathbb{R}^{3}$ centred at $\boldsymbol{x}$ of radius $r>0$ by $B_{\text {ext }}(x, r)$, and its area by $A_{\text {ext }}(x, r)$. Then for all $r>0$, we have

$$
\pi \leq \frac{A_{\mathrm{ext}}(x, r)}{r^{2}}+\frac{1}{4} \int_{B_{\mathrm{ext}}(x, r)}|H|^{2} d \mu .
$$

This type of estimate is from [4], and with these constants (which are each sharp) from [6]. By applying it for fixed, carefully chosen $r>0$, and various centres $x \in \mathcal{M}$, one can derive (1.1).

At the core of the present paper is a refined version of (1.4), which can be considered a companion to Theorem 4.2 in [7]. Given $x \in \mathcal{M}^{m}$, we denote the intrinsic open ball in $\mathcal{M}$ centred at $x$ and of (intrinsic) radius $r>0$ by $B(x, r)$, and its volume by $V(x, r)$. By analogy with [7], when $m \geq 2$ we define, for $R>0$, the maximal function

$$
M(x, R):=\sup _{r \in(0, R]} r^{-\frac{1}{m-1}}[V(x, r)]^{-\frac{m-2}{m-1}} \int_{B(x, r)}|\boldsymbol{H}| d \mu,
$$

and measure collapsedness by

$$
\kappa(x, R):=\inf _{r \in(0, R]} \frac{V(x, r)}{r^{m}} .
$$

Lemma 1.2. For $n>m \geq 2$, suppose that $\mathcal{M}$ is an m-dimensional manifold smoothly immersed in $\mathbb{R}^{n}$, which is complete with respect to the induced metric. Then there exists a constant $\delta>0$ dependent only on $m$ such that for any $x \in \mathcal{M}$ and $R>0$, at least one of the following is true:

(i) $M(x, R)>\delta$;

(ii) $\kappa(x, R)>\delta$.

In the case of surfaces $(m=2)$ in $\mathbb{R}^{n}$, we can set $\delta=\frac{\pi}{8}$.

It is worth extracting the main content of this lemma in the case that $\mathcal{M}$ is a closed surface.

Corollary 1.3. Suppose that $\mathcal{M}^{2}$ is a closed surface immersed in $\mathbb{R}^{n}$, and $x \in \mathcal{M}$. Then

$$
\sup _{r>0} \frac{1}{r} \int_{B(x, r)}|\boldsymbol{H}| d \mu>\frac{\pi}{8} .
$$

Note that for any $\mathcal{M}$, the quantity we are maximizing on the left-hand side of (1.5) is made arbitrarily small by sending $r>0$ to either zero or infinity, but the corollary says that there is always some intermediate $r>0$ where it has a good positive lower bound. 
Acknowledgements. I would like to thank Felix Schulze for valuable input to this work. This paper was started during an extended visit to the Max-Planck-Institut für Gravitationsphysik (Albert-Einstein-Institut) in Golm and the Freie Universität Berlin, and I would like to thank these institutions, Gerhard Huisken and Klaus Ecker for their hospitality. The research was partly supported by an EPSRC Advanced Research Fellowship.

\section{Estimates relating curvature and volume}

In this section we prove Lemma 1.2. The main input to the proof will be the MichaelSimon Sobolev inequality:

Lemma 2.1. In the setting of Lemma 1.2, there exists a constant $\sigma>0$ dependent only on $m$ such that if $f \in W^{1,1}(\mathcal{M})$ then

$$
\sigma\|f\|_{L^{\frac{m}{m-1}}} \leq\|\nabla f\|_{L^{1}}+m\|f \boldsymbol{H}\|_{L^{1}} .
$$

In the case that $m=2$, we may take $\sigma(2)=\sqrt{2 \pi}$.

For the original proof, see [2]. As for the $m=2$ case, we give a proof with the explicit $\sigma(2)$ in the appendix, based on ideas of Leon Simon as we describe there.

Armed with Lemma 2.1, we can prove Lemma 1.2.

Proof. Suppose, with a $\delta>0$ to be chosen later, that $M(x, R) \leq \delta$. Then for all $r \in(0, R]$,

$$
\int_{B(x, r)}|\boldsymbol{H}| d \mu \leq \delta r^{\frac{1}{m-1}}[V(x, r)]^{\frac{m-2}{m-1}} .
$$

Note that for fixed $x, V(r):=V(x, r)$ is a locally Lipschitz function of $r>0$. Indeed, one could locally give an upper bound for the Lipschitz constant in terms of a local lower bound for the Ricci curvature. In particular, $V(r)$ is differentiable for almost all $r>0$. For such $r \in(0, R]$, and any $\mu>0$, define a Lipschitz cut-off function $f$ on $\mathcal{M}$ by

$$
f(y)= \begin{cases}1, & y \in B(x, r), \\ 1-\frac{1}{\mu}\left(\operatorname{dist}_{\mathcal{M}}(x, y)-r\right), & y \in B(x, r+\mu) \backslash B(x, r), \\ 0, & y \notin B(x, r+\mu) .\end{cases}
$$

If we now apply the Michael-Simon estimate from Lemma 2.1, we find that

$$
\sigma V(r)^{\frac{m-1}{m}} \leq \sigma\|f\|_{L^{\frac{m}{m-1}}} \leq \frac{1}{\mu}(V(r+\mu)-V(r))+m\|\boldsymbol{H}\|_{L^{1}(B(x, r+\mu))},
$$


and letting $\mu \downarrow 0$, we deduce that

$$
\sigma V^{\frac{m-1}{m}} \leq \frac{d V}{d r}+m\|\boldsymbol{H}\|_{L^{1}(B(x, r))} .
$$

By (2.1) this gives

$$
\frac{d V}{d r}+m \delta r^{\frac{1}{m-1}} V^{\frac{m-2}{m-1}}-\sigma V^{\frac{m-1}{m}} \geq 0 .
$$

Let us now assume that $\delta>0$ is sufficiently small that $\delta<\omega_{m}$, where $\omega_{m}$ is the volume of the unit ball in $\mathbb{R}^{m}$, and define a function $v(r):=\delta r^{m}$. Computing

$$
\frac{d v}{d r}+m \delta r^{\frac{1}{m-1}} v^{\frac{m-2}{m-1}}-\sigma v^{\frac{m-1}{m}}=\left(m \delta+m \delta^{\frac{2 m-3}{m-1}}-\sigma \delta^{\frac{m-1}{m}}\right) r^{m-1},
$$

we see that by choosing $\delta>0$ sufficiently small, depending only on $m$, we will have (because $m \geq 2$ )

$$
\frac{d v}{d r}+m \delta r^{\frac{1}{m-1}} v^{\frac{m-2}{m-1}}-\sigma v^{\frac{m-1}{m}} \leq 0 .
$$

Note that if $m=2$, because $\sigma(2)=\sqrt{2 \pi}$, we are free to choose $\delta=\frac{\pi}{8}$ in order to satisfy (2.3) and the previous constraint $\delta<\omega_{2}=\pi$. Given (2.2) and (2.3) and the fact that $V(r) / r^{m} \rightarrow \omega_{m}$ as $r \downarrow 0$, while $v(r) / r^{m}=\delta<\omega_{m}$, we deduce that $V(r)>v(r)$ for all $r \in(0, R]$ and hence

$$
\kappa(x, R)=\inf _{r \in(0, R]} \frac{V(x, r)}{r^{m}}>\delta,
$$

as desired.

\section{Diameter control}

We now wish to combine Lemma 1.2 with a covering argument, along the lines of [7], in order to prove Theorem 1.1.

Proof. We may assume that $m \geq 2$ since the case $m=1$ is trivially true with $C(1)=\frac{1}{2}$. Choose $R>0$ sufficiently large so that the total volume of the manifold is less than $\delta R^{m}$, where $\delta$ is given by Lemma 1.2. In particular, for all $z \in \mathcal{M}$, we must have $\kappa(z, R) \leq \frac{V(z, R)}{R^{m}} \leq \delta$, so by Lemma 1.2, we can be sure that $M(z, R)>\delta$. Unravelling the definition of the maximal function, and applying the Hölder inequality, we see that there exists $r=r(z)$ such that

$$
\begin{aligned}
\delta & <r^{-\frac{1}{m-1}} V(z, r)^{-\frac{m-2}{m-1}} \int_{B(z, r)}|\boldsymbol{H}| d \mu \\
& \leq r^{-\frac{1}{m-1}}\left(\int_{B(z, r)}|\boldsymbol{H}|^{m-1} d \mu\right)^{\frac{1}{m-1}},
\end{aligned}
$$


and hence

$$
r(z) \leq \delta^{1-m} \int_{B(z, r(z))}|\boldsymbol{H}|^{m-1} d \mu .
$$

Now let $x, y \in \mathcal{M}$ be any two extremal points, by which we mean that $d_{\text {int }}=$ $\operatorname{dist}_{\mathcal{M}}(x, y)$, and let $\Sigma \subset \mathcal{M}$ be any shortest geodesic connecting $x$ and $y$. The set of balls $B(z, r(z))$ with $z \in \Sigma$ is clearly a covering of $\Sigma$. By a minor perturbation of the covering argument in [7, Lemma 5.2], we know that for any $\lambda \in\left(0, \frac{1}{2}\right)$, we can find a sequence of points $\left\{z_{i}\right\} \subset \Sigma$ such that the balls $\left\{B\left(z_{i}, r\left(z_{i}\right)\right)\right\}$ are disjoint and cover at least a fraction $\lambda$ of the whole of $\Sigma$ :

$$
\lambda d_{\mathrm{int}} \leq \sum_{i} 2 r\left(z_{i}\right)
$$

Combining with (3.2), we may then estimate

$$
\begin{aligned}
d_{\text {int }} & \leq \frac{2}{\lambda} \sum_{i} r\left(z_{i}\right) \leq \frac{2}{\lambda} \delta^{1-m} \sum_{i} \int_{B\left(z_{i}, r\left(z_{i}\right)\right)}|\boldsymbol{H}|^{m-1} d \mu \\
& \leq \frac{2}{\lambda} \delta^{1-m} \int_{\mathcal{M}}|\boldsymbol{H}|^{m-1} d \mu .
\end{aligned}
$$

Since we may take $\lambda$ as close as we like to $\frac{1}{2}$, we arrive at the desired estimate

$$
d_{\text {int }} \leq 4 \delta^{1-m} \int_{\mathcal{M}}|\boldsymbol{H}|^{m-1} d \mu .
$$

Note that in the case $m=2$, we can then give the coefficient $4 \delta^{1-m}$ explicitly as $\frac{32}{\pi}$ since $\delta=\frac{\pi}{8}$ by Lemma 1.2 .

\section{Appendix}

It is a well-known fact, due to but not published by Leon Simon, that the isoperimetric inequality holds on a minimal surface in Euclidean space, with a constant optimal up to a factor of 2. (In conventional notation, this says that $2 \pi A \leq L^{2}$.) It is perhaps less well appreciated that his same proof gives an almost-optimal Sobolev inequality on an arbitrary complete immersed surface, as claimed in Lemma 2.1, and we sketch the proof in this appendix.

First, note that by replacing $f$ by $|f|$, we may assume that $f \geq 0$, and by approximation, we may assume that $f$ is smooth with compact support.

The argument relies on two applications of the first variation formula

$$
\int_{\mathcal{M}} \operatorname{div}_{\mathcal{M}} \Phi=-2 \int_{\mathcal{M}} \Phi \cdot \boldsymbol{H}
$$


where $\Phi$ is a vector field in $\mathbb{R}^{n}$ defined on the surface. (We have adopted the conventions of [6].) One may compute that

$$
\begin{gathered}
\operatorname{div}_{\mathcal{M}} \frac{\boldsymbol{x}}{|\boldsymbol{x}|^{2}}=\frac{2\left|\boldsymbol{x}^{\perp}\right|^{2}}{|\boldsymbol{x}|^{4}} \\
\operatorname{div}_{\mathcal{M}} \frac{\boldsymbol{x}}{|\boldsymbol{x}|}=\frac{1}{|\boldsymbol{x}|}+\frac{\left|\boldsymbol{x}^{\perp}\right|^{2}}{|\boldsymbol{x}|^{3}} \geq \frac{1}{|\boldsymbol{x}|}
\end{gathered}
$$

where $\boldsymbol{x}^{\perp}$ is the projection of the vector $\boldsymbol{x}$ onto the normal space at the point $\boldsymbol{x}$. Consequently, by applying (4.1) first with $\Phi(x)=f(x) \frac{x}{|x|^{2}}$, if the immersion of $\mathcal{M}$ maps over the origin $0 \in \mathbb{R}^{n}$, we have

$-2 \pi f(0)-2 \int_{\mathcal{M}} f \frac{\boldsymbol{x}}{|\boldsymbol{x}|^{2}} \cdot \boldsymbol{H}=\int_{\mathcal{M}} \operatorname{div}_{\mathcal{M}}\left(f \frac{\boldsymbol{x}}{|\boldsymbol{x}|^{2}}\right)=\int_{\mathcal{M}}\left(\nabla f \cdot \frac{\boldsymbol{x}}{|\boldsymbol{x}|^{2}}+2 f \frac{\left|\boldsymbol{x}^{\perp}\right|^{2}}{|\boldsymbol{x}|^{4}}\right)$, and thus

$$
2 \pi f(0) \leq \int_{\mathcal{M}}\left(\frac{|\nabla f|(x)}{|\boldsymbol{x}|}+\frac{2 f(x)|\boldsymbol{H}|(x)}{|\boldsymbol{x}|}\right) d x
$$

Moreover, by applying (4.1) with $\Phi=f(y) \frac{\boldsymbol{y}}{|\boldsymbol{y}|}$, we have

$$
-2 \int_{\mathcal{M}} f \frac{\boldsymbol{y}}{|\boldsymbol{y}|} \cdot \boldsymbol{H}=\int_{\mathcal{M}} \operatorname{div}_{\mathcal{M}}\left(f \frac{\boldsymbol{y}}{|\boldsymbol{y}|}\right)=\int_{\mathcal{M}}\left(\frac{\boldsymbol{y} \cdot \nabla f}{|\boldsymbol{y}|}+f \operatorname{div}_{\mathcal{M}} \frac{\boldsymbol{y}}{|\boldsymbol{y}|}\right)
$$

and thus by (4.3) (with $\boldsymbol{x}$ replaced by $\boldsymbol{y}$ ) we find that

$$
\int_{\mathcal{M}} \frac{f(y)}{|\boldsymbol{y}|} d y \leq \int_{\mathcal{M}}(|\nabla f|+2 f|\boldsymbol{H}|) .
$$

Returning to (4.4) with the origin translated to an arbitrary point $\boldsymbol{y} \in \mathbb{R}^{n}$ on the surface, then multiplying by $f(y)$ and integrating, we have

$$
\begin{aligned}
\int_{\mathcal{M}} 2 \pi f^{2}(y) d y & \leq \int_{\mathcal{M}}\left[\int_{\mathcal{M}}\left(\frac{|\nabla f|(x)}{|\boldsymbol{x}-\boldsymbol{y}|}+\frac{2 f(x)|\boldsymbol{H}|(x)}{|\boldsymbol{x}-\boldsymbol{y}|}\right) d x\right] f(y) d y \\
& =\int_{\mathcal{M}}(|\nabla f|(x)+2 f(x)|\boldsymbol{H}|(x))\left(\int_{\mathcal{M}} \frac{f(y)}{|\boldsymbol{x}-\boldsymbol{y}|} d y\right) d x .
\end{aligned}
$$

This we can simplify using (4.5), this time with the origin translated to the point $\boldsymbol{x}$, to give

$$
\begin{array}{rl}
\int_{\mathcal{M}} & 2 \pi f^{2}(y) d y \\
\leq & \int_{\mathcal{M}}(|\nabla f|(x)+2 f(x)|\boldsymbol{H}|(x))\left(\int_{\mathcal{M}}(|\nabla f|(y)+2 f(y)|\boldsymbol{H}|(y)) d y\right) d x \\
= & \left(\int_{\mathcal{M}}|\nabla f|+2 f|\boldsymbol{H}|\right)^{2},
\end{array}
$$

which completes the proof. 


\section{References}

[1] E. Kuwert and R. Schätzle, Removability of point singularities of Willmore surfaces. Ann. of Math. 160 (2004), 315-357. Zbl 1078.53007 MR 2119722

[2] J. H. Michael and L. M. Simon, Sobolev and mean-value inequalities on generalized submanifolds of $R^{n}$. Comm. Pure Appl. Math. 26 (1973), 361-379. Zbl 0256.53006 MR 0344978

[3] G. Perelman The entropy formula for the Ricci flow and its geometric applications. Preprint 2002. arXiv:math.DG/0211159v1

[4] L. M. Simon, Existence of surfaces minimizing the Willmore functional. Comm. Anal. Geom. 1 (2) (1993), 281-326. Zbl 0848.58012 MR 1243525

[5] P. M. Topping, The optimal constant in Wente's $L^{\infty}$ estimate. Comment. Math. Helv. 72 (1997), 316-328. Zbl 0892.35030 MR 1470094

[6] P. M. Topping, Mean curvature flow and geometric inequalities. J. Reine Angew. Math. 503 (1998), 47-61. Zbl 0909.53044 MR 1650335

[7] P. M. Topping, Diameter control under Ricci flow. Comm. Anal. Geom. 13 (2005), 1039-1055. Zbl 05135035 MR 2216151

[8] P. M. Topping, Lectures on the Ricci flow. London Math. Soc. Lecture Notes Ser. 325, Cambridge University Press, Cambridge 2006. Zbl 1105.58013 MR 2265040 http://www.warwick.ac.uk/ maseq/RFnotes.html

Received September 11, 2006

Peter Topping, Mathematics Institute, University of Warwick, Coventry, CV4 7AL, UK

E-mail: P.M.Topping@warwick.ac.uk 